\title{
Electron Pair in Low Lying Excited States in a Semimagnetic Quantum Dot
}

\author{
K.Mari Raj, R. Muneesvaran, N.G. Prasanna and M.S.S.Saravanakumaar \\ Department of Physics, Saraswathi Narayanan College(Autonomous), Madurai -625022, India
}

\begin{abstract}
Using the method of few-body physics, the solutions of ground state and some low-lying excited states[such as $1 s-1 s, 1 s-1 p$ and $1 s-1 d$ ] of electron pair in a semimagnetic quantum dot have been obtained. The dependence of the confined energies of the electron pair in a semimagnetic quantum dot is studied as a function of dot radius, different barrier height and inter electron distances. Our results are good in agreement with existing literatures.
\end{abstract}

Keywords: Confined energy, semi magnetic quantum dot, variation principle

\section{Introduction}

Quantum dots - "an artificial atom" in which only a few electrons are bound at semiconductor interfaces have been the subject of intense research studies over the last few decades. The electron motion in quantum dots are confined to a region with dimensions comparable to the de Broglie wavelength of the particle which results the quantization of energy. The intensive investigations on semiconductor quantum dots have revealed that optoelectronic properties of such systems are quite sensitive to the reduction of their dimensionality and to the strength of applied external magnetic field, and depend strongly on the electronelectron interaction[1-3]. Diluted magnetic semiconductors (DMSs) or Semimagnetic Quantum Structures are expected to play an important role in interdisciplinary materials science and future electronics because charge and spin degrees of freedom accommodated into a single material exhibit interesting magnetic, magnetooptical, magnetoelectronic, and other properties[4]. Study of DMSs and their heterostructures have centered mostly on II-VI semiconductors, such as CdTe and $\mathrm{ZnSe}$, in which the valence of the cations matches that of the common magnetic ions such as Mn.

Bryant [5] first reported that the role of two electrons in a cubical quantum dot and showed that there is a continuous evolution of the energy level structure, from the single - particle like states in the limit of a small dot, to a level structure in larger dots where electron - electron interactions are dominant thus kindling the interest in impurity states. The double electron states in parabolic quantum well, taking into account the nonparabolicity of the conduction band have been reported by E.C, Niculescu[6]. A.John Peter and S.Saravanakumar [7] calculated the interaction energy of two electrons in low lying states in a GaAs systems. Coulomb interaction energy of a double acceptor impurity in a semimagnetic quantum dot have been studied in $\operatorname{Ref}[8]$.

In this work, we present the calculation of columbic interaction energy of two electrons in a $\mathrm{Cd}_{1-}$ ${ }_{x} \mathrm{Mn}_{\mathrm{x}} \mathrm{Te} / \mathrm{CdTe}$ quantum dot in the effective mass approximation using variational principle. The two electron problem is also treated as an effective one body problem with the Hamiltonian. The models and calculations are provided in Section I, while the results and discussion are given in Section II.

\section{Model And Theory}

Within the framework of effective mass approximation, the total Hamiltonian for two electron in CdTe quantum dot is given by

$$
\mathrm{H}=\mathrm{H}_{0}+\mathrm{H}^{\prime}
$$

$$
\begin{array}{r}
H_{0}=\sum_{j=1}^{2}\left\{\frac{\bar{p}_{j}^{2}}{2 m^{*}}+V_{D}\left(\bar{r}_{j}\right)\right\} \\
H^{\prime}=\frac{e^{2}}{\varepsilon_{0}\left|\overline{r_{1}}-\overline{r_{2}}\right|}
\end{array}
$$


where $V_{D}(\bar{r})$ is the barrier height given by $V_{D}(\bar{r})=Q_{c} \Delta E_{g}(x) . \mathrm{Q}^{\mathrm{c}}$ is the conduction band off-set parameter, which is taken to be 0.658 and the band gap difference between $\mathrm{CdTe}$ and $\mathrm{Cd}^{1-\mathrm{x}} \mathrm{Mn}^{\mathrm{x}} \mathrm{Te}$ is given by $[9,10]$

$$
\Delta E_{g}(x)=0.11(1-x)+0.177 x \quad e V .
$$

The second term H' is the Coulomb interaction energy, we treat as perturbation which is calculated numerically here. The units of length and energy used throughout the present paper are the effective Bohr radius $R^{*}=\hbar^{2} \varepsilon_{o} / m^{*} e^{2}$ and $R_{y}^{*}=m^{*} e^{4} / 2 \varepsilon_{o}^{2} \hbar^{2}$ where $\varepsilon_{o}$ is the static dielectric constant of CdTe and $\mathrm{m}^{*}$ is the effective mass of electron.

The eigen functions for the three lowest lying states within the dot are given by

$$
\psi_{1 s}(\bar{r})= \begin{cases}N_{1} \frac{\sin \left(\alpha_{1} r\right)}{r}, & r<R, \\ N_{2} \frac{e^{-\beta_{1} r}}{r}, & r \geq R\end{cases}
$$

$$
\psi_{1 p}(\bar{r})= \begin{cases}N_{3}\left[\frac{\sin \left(\alpha_{2} r\right)}{\left(\alpha_{2} r\right)^{2}}-\frac{\cos \left(\alpha_{2} r\right)}{\alpha_{2} r}\right] \cos \theta, & r<R, \\ N_{4}\left[\frac{1}{\beta_{2} r}+\frac{1}{\left(\beta_{2} r\right)^{2}}\right] e^{-\beta_{2} r} \cos \theta, & r \geq R\end{cases}
$$

$$
\psi_{1 d}(\bar{r})=\left\{\begin{array}{l}
N_{5}\left[\left(\frac{3}{\left(\alpha_{3} r\right)^{3}}-\frac{1}{\alpha_{3} r}\right) \sin \left(\alpha_{3} r\right)-\frac{3}{\left(\alpha_{3} r\right)^{2}} \cos \left(\alpha_{3} r\right)\right]\left(3 \cos ^{2} \theta-1\right) \\
N_{6}\left[\frac{1}{\beta_{3} r}+\frac{1}{\left(\beta_{3} r\right)^{2}}+\frac{1}{\left(\beta_{3} r\right)^{3}}\right] e^{-\beta_{3} r\left(3 \cos ^{2} \theta-1\right)} \quad r<R,
\end{array}\right.
$$

where $\mathrm{N}_{1}, \mathrm{~N}_{2}, \mathrm{~N}_{3}, \mathrm{~N}_{4}, \mathrm{~N}_{5}$ and $\mathrm{N}_{6}$ are normalization constants $\alpha_{1}$ and $\beta_{1}$ are given by $\alpha_{1}=\sqrt{2 m^{*} E_{1}}$ and $\beta_{1}=\sqrt{2 m^{*}\left(V_{D}-E_{1}\right)}$.

Matching the wave functions and their derivatives at the boundary $r=R$, the energy eigen values are determined by imposing the boundary conditions, 


$$
\left.\frac{i \hbar}{m^{*}} \frac{\partial \psi}{\partial r}(r<R)\right|_{r=R}=\left.\frac{i \hbar}{m^{*}} \frac{\partial \psi}{\partial r}(r \geq R)\right|_{r=R}
$$

Using Eqs.(5) -(9), we obtain

$$
\begin{gathered}
\alpha_{1} R+\beta_{1} R \tan \left(\alpha_{1} R\right)=0 \text { for s-states } \\
\frac{\cot \left(\alpha_{2} R\right)}{\alpha_{2} R}-\frac{1}{\left(\alpha_{2} R\right)^{2}}=\frac{1}{\beta_{2} R}+\frac{1}{\left(\beta_{2} R\right)^{2}} \text { for p-states } \\
9 \alpha_{3} R-\left(\alpha_{3} R\right)^{3}+\left\lfloor 4\left(\alpha_{3} R\right)^{2}-9\right] \tan \left(\alpha_{3} R\right)=-\left[\left(3-\left(\alpha_{3} R\right)^{2} \tan \left(\alpha_{3} R\right)-3\left(\alpha_{3} R\right)\right]\right.
\end{gathered}
$$

$$
\left[\frac{\left(\beta_{3} R\right)^{3}+4\left(\beta_{3} R\right)^{2}+9 \beta_{3} R+9}{\left(\beta_{3} R\right)^{2}+3 \beta_{3} R+3}\right] \text { for } d \text { states }
$$

Solving these transcendental equations, the confined particle energies

$E_{l}^{(n)}(n=1,2,3, \ldots \ldots, l=0,1)$

$=2,3 \ldots$. The confinement energies of the first three states are drawn .

Considering s-states, p-states and d-states, the triplet state energies are obtained using the wave functions $\Psi_{1 \mathrm{~s}-1 \mathrm{~s}}(\mathrm{r} 1, \mathrm{r} 2), \Psi_{1 \mathrm{~s}-1 \mathrm{p}}(\mathrm{r} 1, \mathrm{r} 2)$ and $\Psi_{1 \mathrm{~s}-1 \mathrm{~d}}(\mathrm{r} 1, \mathrm{r} 2)$ as expressed above.

The confining potential energies of two interacting electrons are calculated using the Hamiltonian

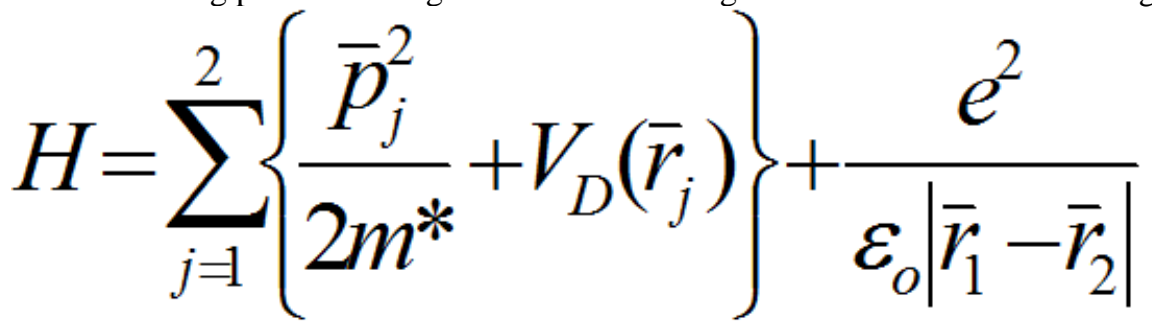

and the wave functions Eqs.(5)-(7) for different states of s,p and d symmetry.Using eqn (8),we have obtained the non-interacting and interacting two electron system numerically and the results are drawn.

\section{Results And Discussion}

Our numerical computation is carried out for one of the typical semi magnetic materials, CdTe, as an example with the material parameters shown in the following: $\varepsilon_{0}=9.6$ and $\mathrm{m}^{*}=0.11 \mathrm{~m}_{\mathrm{o}}$ where $\mathrm{m}_{\mathrm{o}}$ the single electron bare mass. the potential well depth is taken as $\mathrm{V}=4.9996 \mathrm{Ry}$ for $\mathrm{x}=0.2$ and $5.5427 \mathrm{Ry}$ for $\mathrm{x}=0.4$ and 6.0856 Ry for $\mathrm{x}=0.6$ respectively, with $\mathrm{R}_{\mathrm{y}}=16.24 \mathrm{meV}$ and $\mathrm{R}^{*}=46.18 \AA$ Á.

Fig.2.1 shows the variation of confined energies of $1 \mathrm{~s}-1 \mathrm{~s}$ states with the dot radius for three different concentrations as a function of inter electron distances with and without the interaction energy. We have estimated the Coulomb interaction energy numerically. We find that as the dot size increases the confinement energy of the electron decreases for all the cases as expected. Confined electron energies are always higher for higher concentration for all the dot radii. Coulombic interaction energy of two electron is appreciable for inter electron distance is $50 \mathrm{~A}^{\mathrm{o}}$ and decreases when inter electron distance increases and shifting towards the confined energy of effective one body problem in all cases. It shows that the correlation effects are important for smaller dots and should be considered in the studies on low dimensional semiconducting systems. There is no appreicable change in correlation energy for larger dot sizes. As the value of the barrier height is larger compared to than that of correlation energy, the increase of barrier height has no impact on correlation energy[7].

Fig 2.2. shows the variation of $1 \mathrm{~s}-1 \mathrm{p}$ confined energies as a function of dot radius for inter-electron distance with different Mn concentration. We find that as the dot size decreases confinement energy of two electron system increases up to the radius $\boldsymbol{R} \leq \mathbf{3 0} \AA$ above which one cannot expect the bound states for the three different Mn concentrations. Unbound states exist when dot radius is reduced below $30 \AA$. The play of inter electron distance and concentration is unappreciable for $1 \mathrm{~s}-1 \mathrm{p}$ states.

We present the variation of $1 \mathrm{~s}-1 \mathrm{~d}$ confined energies as a fuunction of dot radius for various interelectron distances with differenrent concentrations in Fig 2.3. Confinement energy increases with 
reduction of dot sizes for all the Mn concentrations. Confined electron energies are always higher for higher concentration for all the dot radii. Coulombic interaction energy of two electron is appreciable for inter electron distance is 50 Ao because effective Bohr radius for CdTe quantum dot is $\mathrm{R}^{*}=46.18 \AA$ and decreases when inter electron distance increases and shifting towards the confined energy of effective one body problem in all cases. The confined energy of $1 \mathrm{~s}-1 \mathrm{~s}$ two electron systems are always higher than other two interactions of $1 \mathrm{~s}-1 \mathrm{p}$ and $1 \mathrm{~s}-1 \mathrm{~d}$ symmetry for any dot size.
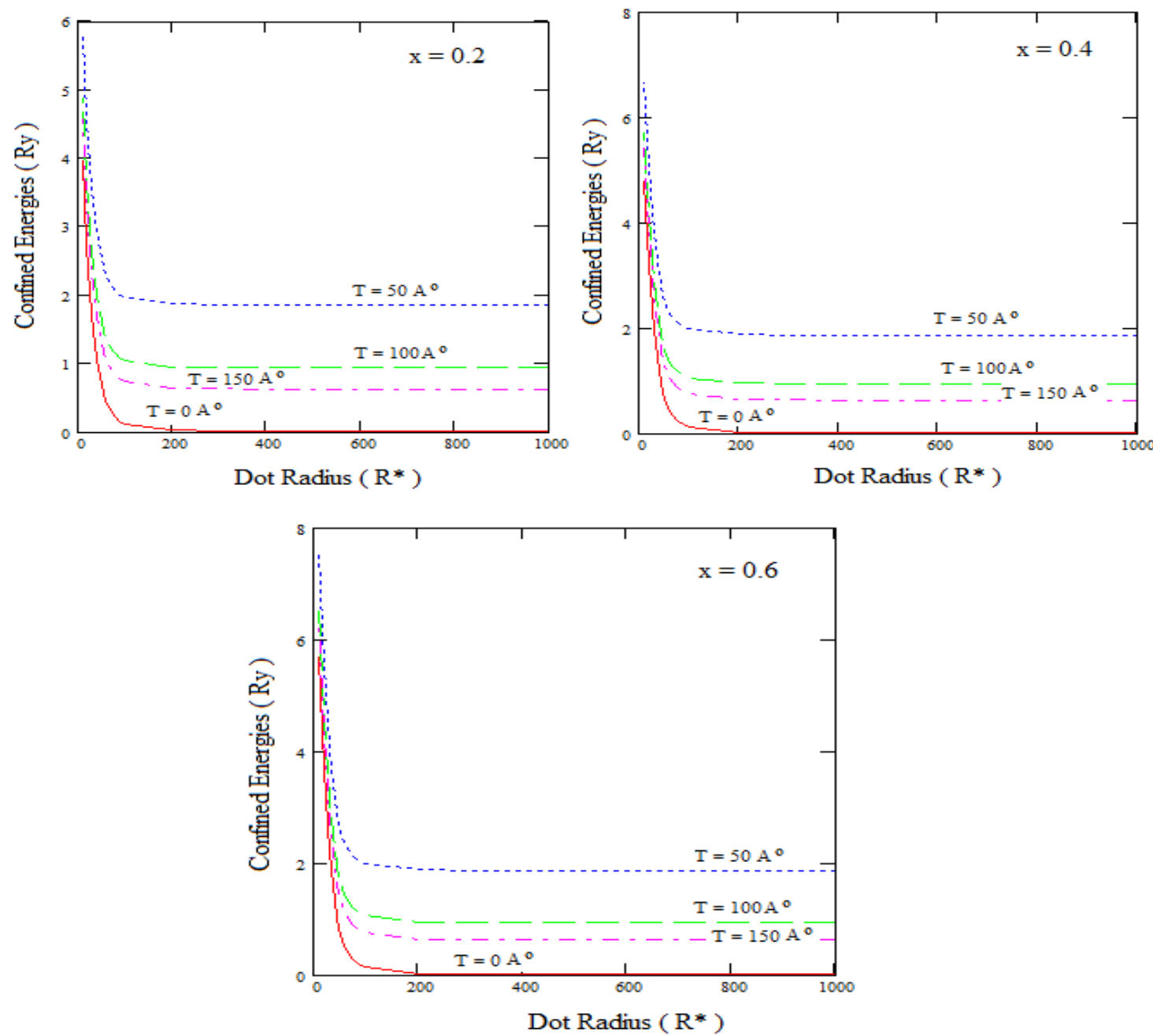

Fig 2.1 Variation of 1s- 1s confined energies as a function of dot radius for various inter electron distance with different Mn concentration. 

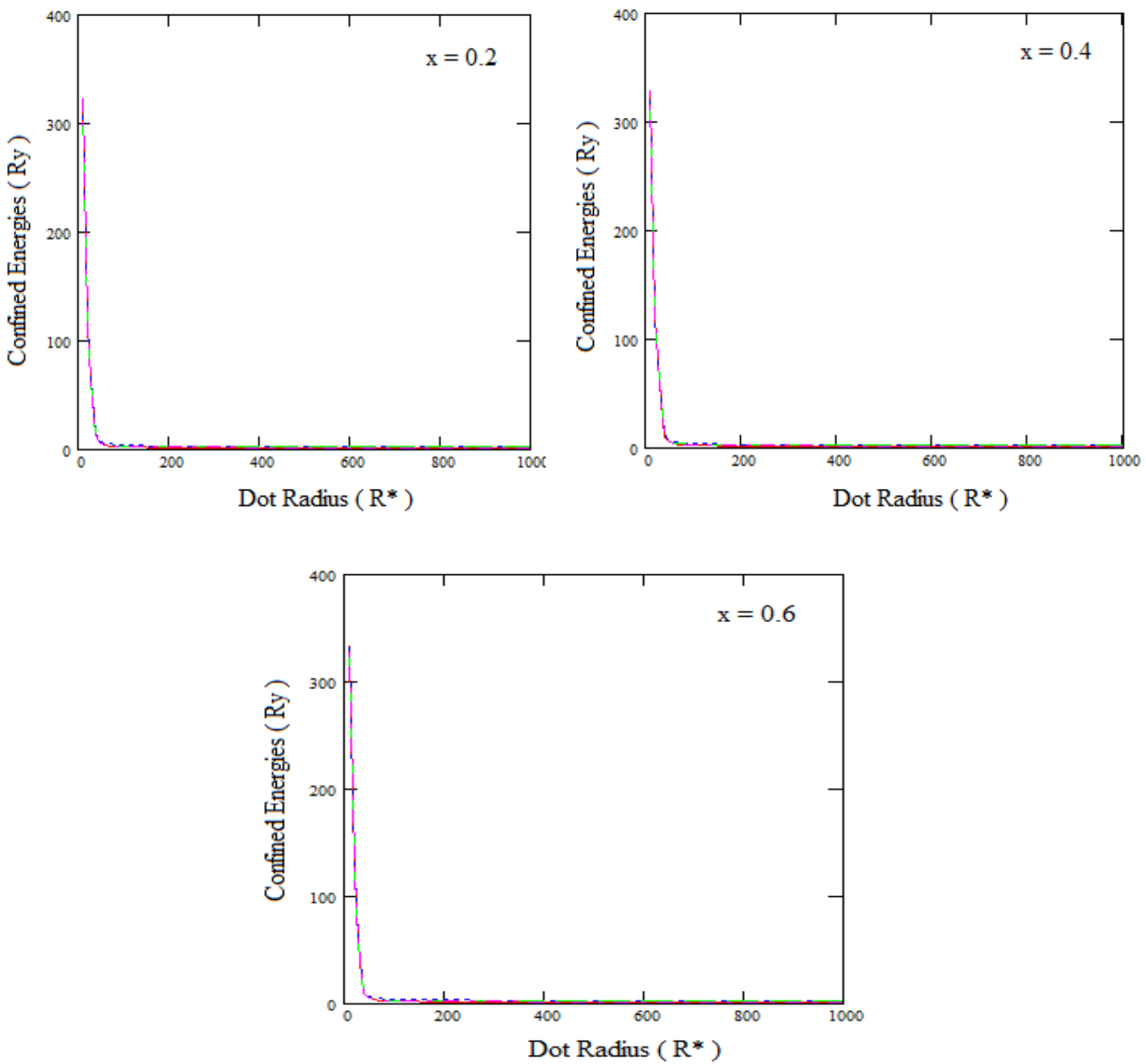

Fig 2.2. Variation of $1 \mathrm{~s}-1 \mathrm{p}$ confined energies as a function of dot radius for various inter electron distance with different Mn concentration.
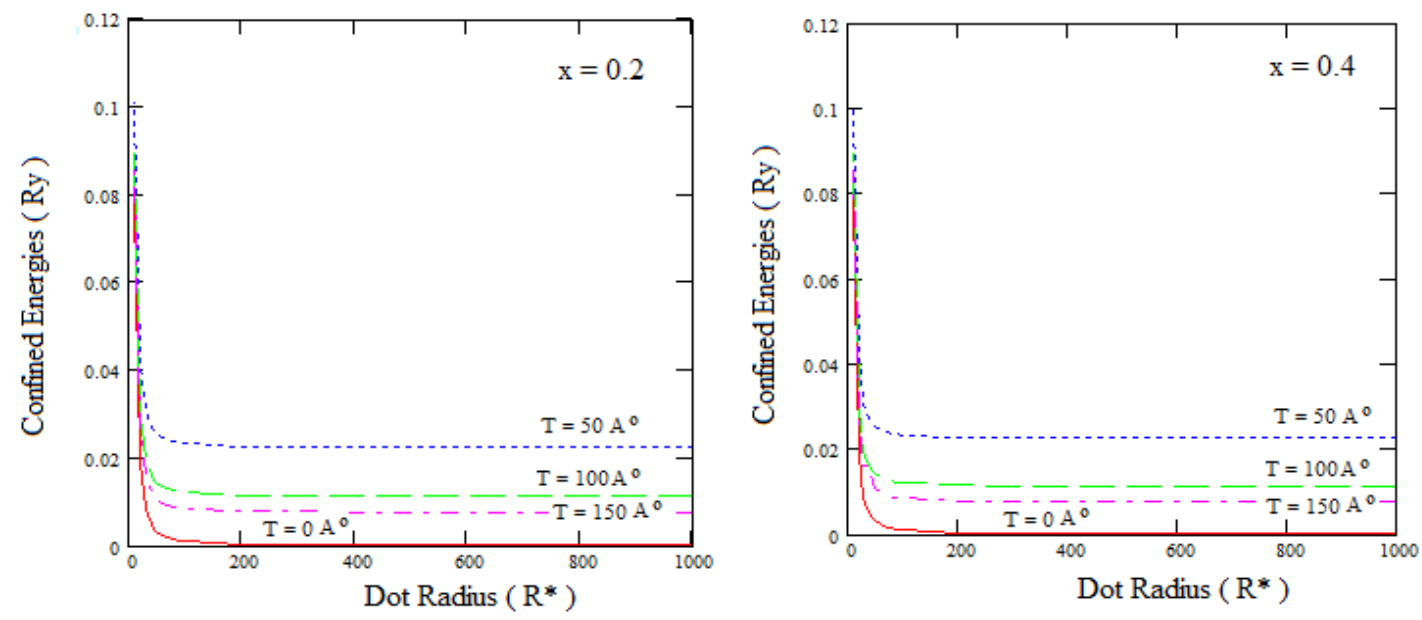

National Conference on Current Advancements in Physics $3^{\text {rd }} \& 4^{\text {th }}$ February 2017 


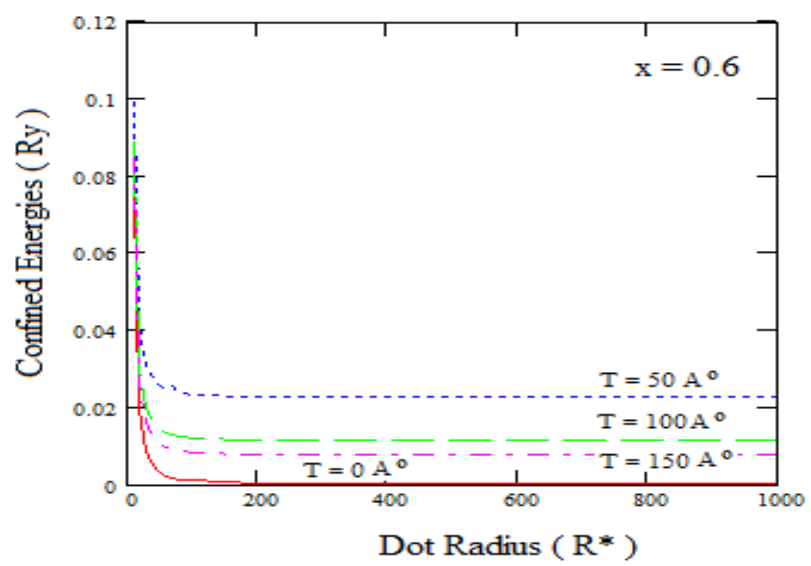

Fig 2.3 Variation of 1s- 1d confined energies as a function of dot radius for various inter electron distance with different Mn concentration.

\section{Conclusion}

We have calculated the coulmbic interaction energies of two electrons in ground state and low lying excited states for different concentrations of $\mathrm{Mn}$ ions for the finite dot of $\mathrm{Cd}_{1-\mathrm{x}} \mathrm{Mn}_{\mathrm{x}} \mathrm{Te}$ for various inter electron distances using a variational approach. The main results are the confined energy of single electron in ground state increases with increase of $\mathrm{Mn}$ concentrations. For low lying excited states, increase of concentration of $\mathrm{Mn}$ ions seems to be insignificant.The coulomb interaction energy is very significant contributing of the confinement energy for smaller dots in and the interaction energy is appreciable for inter electron distance is below the effective bohr radius $R<=50 \mathrm{~A}^{\circ}$.The barrier height has no effect on interaction of electrons as their contribution is small compared to that of barrier height.However, this study could shed some light on the study of many body systems in semimagnetic quantum structures.

\section{References}

[1]. Kandemir ,B S , Variational study of two-electron quantum dots,Phys. Rev. B 72165350, ,2005)1-6.

[2]. Sato, K. et al. First-principles theory of dilute magnetic semiconductors. Rev.Mod.Phys.82, (2010).1633-1690.

[3]. B. Szafran, J. Adamowski, S. Bednarek, Electron-electroncorrelationinquantumdots, PhysicaE5(2000)185-195.

[4]. Sato,K.,Dederichs,P.H.,Katayama-Yoshida,H.\&Kudrnovsky,J,Exchange interactionsindilutedmagneticsemiconductors.J.Phys.Condens.Matter 16 (2004) S5491-S5497.

[5]. George W. Bryant, Electronic Structure of Ultrasmall Quantum-Well Boxes Phys. Rev. Lett. 59 (1987) 1140-1143.

[6]. E.C. Niculescu, Binding energy of a double donor in a parabolic quantum well, Phys.Lett. A 197(1995)330 - 334.

[7]. John Peter, S.Saravanakumar,Two interacting electrons in low lying states in a spherical quantum dot, Physica E 41 (2008) 138-141.

[8]. A.M. J. D Reuben, K.Jayakumar, Coulomb interaction energy of double acceptor in a semi magnetic quantum dot,Superlattices and Microstructres 52(2012)732-737.

[9]. J.R. Buschert, F.C. Peiris, N. Samarth, H.Luo, J.K. Furdyna, Unusual elastic constants of cubic MnTe in strainedlayer superlattices measured by x-ray diffraction ,Phys. Rev. B49(1994)4619-4622

[10]. Eunsoon oh, C. Parks, I. Miotkowski, M.Dean Sciacca, A.J. Mayur, A.K. Ramdas, Optical properties of Mg-based II-VI ternaries and quaternaries: Cd1-xMgxTe and Cd1-x-yMgxMnyTe, Phys. Rev. B48(1993)15040-15046. 